\title{
HiCmapTools: a tool to access HiC contact maps
}

\author{
Jia-Ming Chang ${ }^{1 *} \mathbb{D}$, Yi-Fu Weng ${ }^{1}$, Wei-Ting Chang ${ }^{1}$, Fu-An Lin $^{1}$ and Giacomo Cavalli²
}

\author{
${ }^{*}$ Correspondence: \\ chang.jiaming@gmail.com \\ ${ }^{1}$ Department of Computer \\ Science, National Chengchi \\ University, 11605 Taipei City, \\ Taiwan \\ Full list of author information \\ is available at the end of the \\ article
}

\begin{abstract}
Background: With the development of HiC technology, more and more HiC sequencing data have been produced. Although there are dozens of packages that can turn sequencing data into contact maps, there is no appropriate tool to query contact maps in order to extract biological information from $\mathrm{HiC}$ datasets.

Results: We present HiCmapTools, a tool for biologists to efficiently calculate and analyze HiC maps. The complete program provides multi-query modes and analysis tools. We have validated its utility on two real biological questions: TAD loop and TAD intra-density.

Conclusions: HiCmapTools supports seven access options so that biologists can quantify contact frequency of the interest sites. The tool has been implemented in $\mathrm{C}++$ and $R$ and is freely available at https://github.com/changlabtw/hicmaptools and documented at https://hicmaptools.readthedocs.io/.
\end{abstract}

Keywords: Hi-C, Topologically Associating Domains (TADs), 3D genome, Juicer, hicpipe

\section{Background}

With the invention of the microscope, researchers gained a preliminary understanding of the chromosome's tertiary structure. However, it was difficult to gain a more global picture, that is, until the development of chromosome conformation capture (3C) [1] and its variations $4 \mathrm{C}$ [2], $5 \mathrm{C}$ [3], and $\mathrm{HiC}$ [4], which have made available spatial information on the whole genome. There are now many $\mathrm{HiC}$ pipelines available [5, 6]. However, there is no suitable tool to access $\mathrm{HiC}$ map results except for visualization (Table 1), that is, no systematic way to extract $\mathrm{HiC}$ contact information for a specific query. For example, given the list of CTCF binding sites, a custom script is needed to compute all pairwise contacts between them. Therefore, we have developed HiCmapTools, which helps biologists efficiently query $\mathrm{HiC}$ maps and perform permutation tests. It supports seven query modes and attempts to cover the most frequent needs of biologists who use HiC to study chromatin contacts and their putative function. 
Table 1 Comparison between HiCmapTools and other current tools applied to HiC sequencing datasets

\begin{tabular}{lllll}
\hline Function & HiCmapTools & HiCPro [7] & $\begin{array}{l}\text { Juicer [8], } \\
\text { Juicebox [9] }\end{array}$ & $\begin{array}{l}\text { gcMapExplorer } \\
\text { [10] }\end{array}$ \\
\hline Generate HiC map & $x$ & 0 & 0 & $\times$ \\
Visualization & $x$ & $x$ & 0 & 0 \\
Format transformation & $x$ & 0 & 0 & 0 \\
Extra submap & 0 & $x$ & dump & $x$ \\
Query HiC map & 0 & MAKE_VIEWPOINTS.PY* & $x$ & $x$ \\
\hline
\end{tabular}

* Generates a BED profile from a specified viewpoint (similar to the -bait query mode of HicmapTools)

\section{Implementation}

HiCmapTools is implemented in $\mathrm{C}++$, which facilitates using common programming data structures and functions from the Standard Template Library (STL). Users input $\mathrm{HiC}$ maps in either hic format generated by Juicer [8] or bin-contact pair files following hicpipe $[11,12]$. The input contact map is stored as a hash structure using pair bins as keys. The size of the bin is specified by the user (-in_hic_resol for .hic) or depends on the input file (bin-contact pair files). A query is binned into a corresponding key based on its position to facilitate efficient extraction of contact frequency via STL hash operation $(O(1)$ for lookup). Also, we measure the significance of the extracted frequencies using permutation tests which rank the frequency among random samples. The usage of the query mode and random test are explained below.

\section{Query mode}

We use seven query modes to meet the needs of biologists, as illustrated in Fig. 1. The query input is expected to be in BED format, in which each line is considered as an individual query. Sample query files are available at https://hicmaptools.readthedocs.io/en/ latest/format.html\#query-file.

1. bait: calculate average contacts from downstream to upstream (controlled by - ner bin) of a position of interest (white rectangle). For example, biologists can measure the average contact frequency around a PRE binding site.

2. local: list all contacts inside an interval (white cross). All contacts inside a gene body can be extracted by querying specific gene loci.

3. loop: contact frequency between two ends of a loop. As an example query, biologists can test whether gene looping exists [13] by calculating the contact frequency of its promoter with the transcription termination site. A gene of interest is listed as one row in a BED file.

4. pair: contacts between a pair of regions (contact between regions $X$ and $Y$, white crosses). For instance, contact frequencies between a gene promoter and an enhancer are extracted by querying their positions.

5. sites: contacts between specific sites (contacts between three sites, including diagonal). As an example, given the list of chromatin insulator sites, HiCmapTools calculates all pairwise contacts among these sites, such that users can check whether any pair of binding sites interact with each other. 


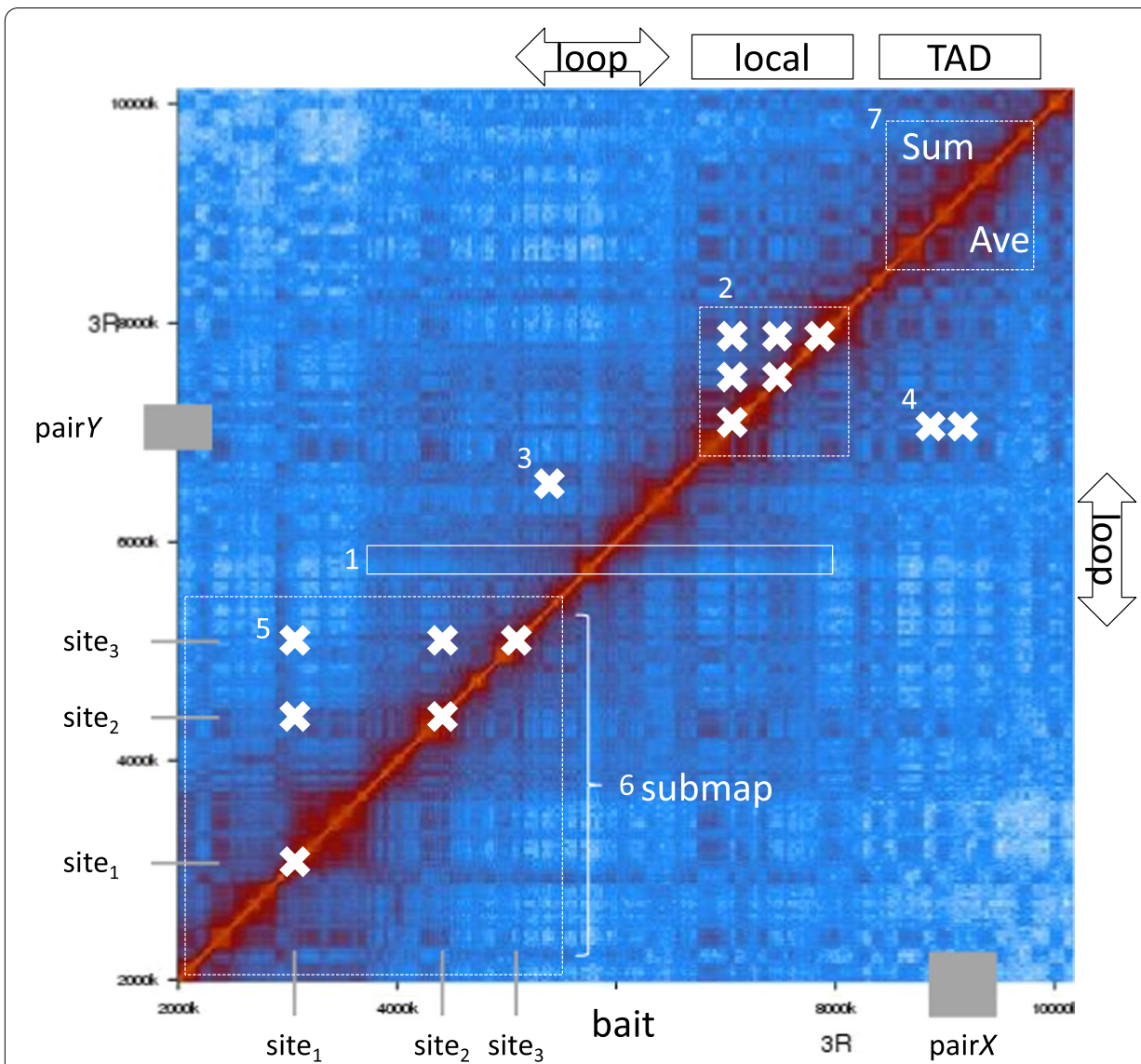

Fig. 1 Illustration of query modes. Numbers indicate the corresponding query modes. HiC data is Drosophila chr3R:2000k..10000k [14]

6. submap: sub contact map of regions of interest. The HiC map is stored efficiently by keeping only selected regions (i.e., a region containing long-range contacts between two loci such as the Drosophila Antp-C and the BX-C).

7. TAD: sum and average of contacts within specific TAD regions (white dashed square at the top right of Fig. 1). Biologists can quantify chromatin compaction within a TAD by measuring the average intra-TAD contact frequency. This might be used to compare different TADs of interest.

\section{Permutation test}

The biological meaning of the extracted contact frequency is assessed by the probability that a given frequency occurs by chance. We approximate the distribution of the null hypothesis by shuffling query positions. That is, we generate alternative queries by randomly shifting the query loci around the same chromosome multiple times (default $=100$ times, controlled by the -random option). Then, we perform the same query mode for the alternative queries and calculate their contact frequencies. Finally, the query's contact frequency is evaluated as its ranking among the sampled frequencies. 


\section{bait query example}

Here, one is interested in whether there is local contact enrichment around specific loci, such as the Ubx gene in the BX-C locus. Then, we perform a -bait query where a $30 \mathrm{k}$ map is used, up/downstream is $150 \mathrm{~kb}(=30 \mathrm{k}$ x $5 \mathrm{bins})$ with 100 permutation tests.

hicmaptools -in_map fly_30k.n_contact -in_bin fly_30k.cbins -bait Ubx.bed -near_bin 5 -random 100 -output Ubxt-bait.tsv

Two outputs are produced: Ubxt-bait.tsv and Ubxt-bait_random_1.txt. The former contains the contact frequency of the query (for example "7237.85") and the average of the sampled frequencies (for example "6363.51") with comparison provided as ratio (for example "1.14") and rank (for example "top 19\%"). The latter provides each sampled frequency, including that of the query (the second line), where the suffix "_1" indicates one query entry in the input file. Output details are available online at https://hicmaptools.readthedocs.io/en/latest/format.html\#output. We provide tools/ visualPermutationTest. $R$, a $R$ script to visualize the query's output against the distribution of the random samples (Fig. 2).

$$
\text { Rscript visualpermutationTest.R Ubxt-bait_random_1.txt }
$$
UbxPermuTest.pdf

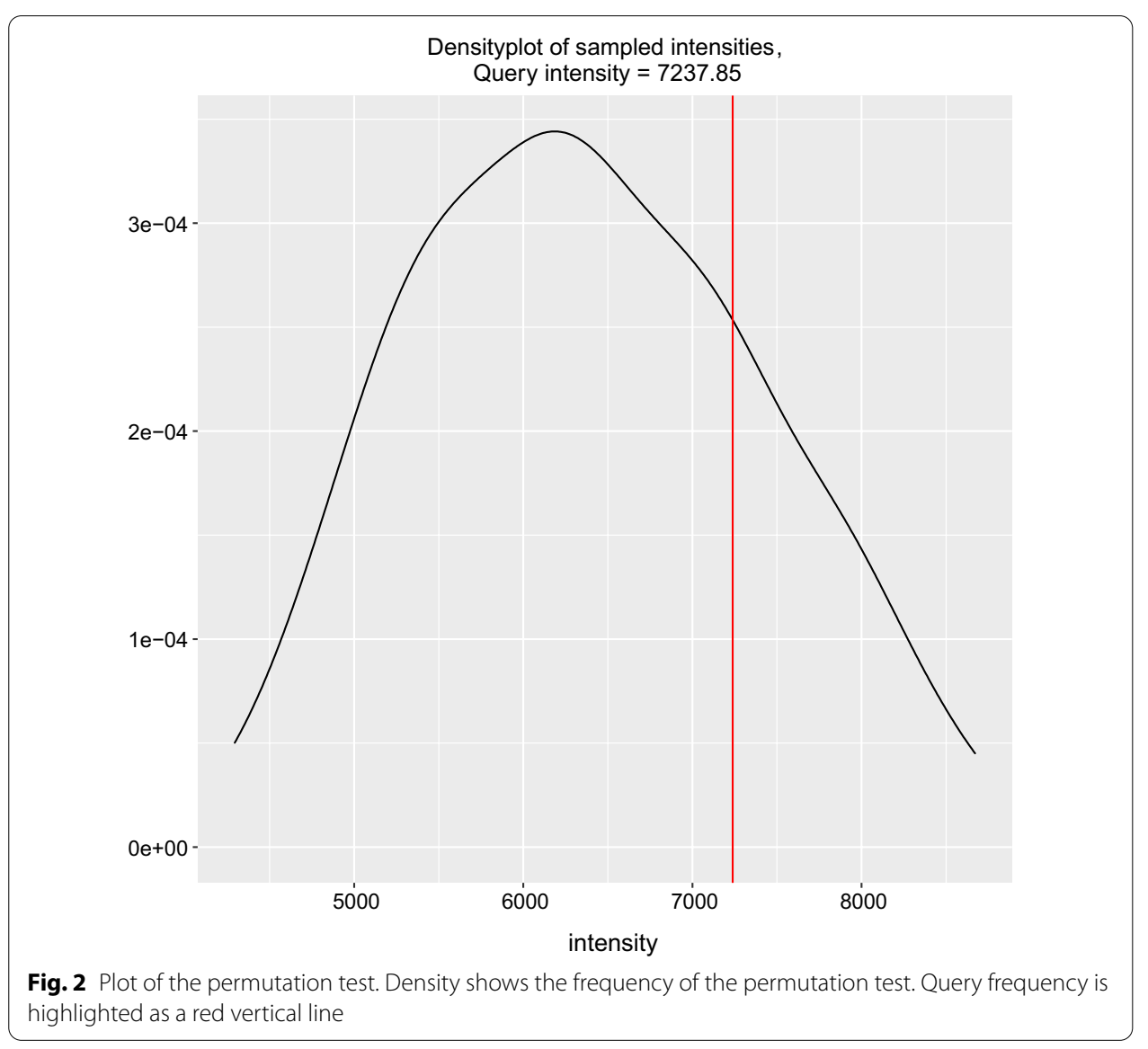


Besides Drosophila, HiCmapTools could handle other species. We test whether there is local contact enrichment around the Tox gene in mouse T Cell. The HiC map, DO_mm10.hic, is available at https://doi.org/10.6084/m9.figshare.18586106 instead of GitHub due to the size limit.

hicmaptools -in_hic D0_mm10.hic -bait Tox_mm10.bed -random

0 -output Tox_bait_Do.txt

\section{Results}

HiC data has shown chromosome structure to be formed by 3D folding of a higher-order unit, the topologically associating domain (TAD) [14-16]. In Drosophila, TADs can be further classified into four epiTADs-active, null, PcG, and HP1-based on ChIP-seq binding profiles [14]. Although a substantial proportion of TADs form loops, with loop bases frequently located at the TAD boundaries in mammals $[17,18]$, few examples of TAD loop structure have been reported in the fly genome [19]. These loops generally involve Polycomb binding sites, but whether any preference exists regarding epiTADs has not been systematically addressed. We selected two related experiments to determine whether HiCmapTools can help biologists address these questions.

We approached the question by applying HiCmapTools on high-resolution Drosophila $\mathrm{HiC}$ data (1 kb, merged embryo from GSE 34,453, 61,471, 94,115, and 99,105, "Availability of data and materials"). There are 1257 TADs with 548 active, 469 null, 136 PcG, and 104 HP1 TADs (Additional file 1).

\section{TAD loop}

We calculate the contact frequency between two boundaries of the TADs via the loop query mode:

hicmaptools -in_map fly.bimap -in_bin fly.bins -loop epiTAD. bed -output resLoop.tsv

Interestingly, PcG TADs show strong loop frequency, whereas active TADs have the lowest frequency (Fig. 3, raw data in Additional file 2). Therefore, we assume that PcG TADs form a loop structure with high probability [20].

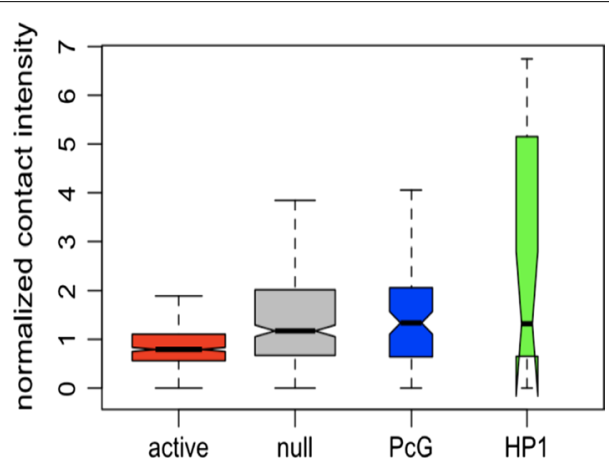

Fig. 3 Boxplot of loop frequency regarding epiTADs 


\section{TAD intra-density}

Furthermore, we check whether intra-TAD contact frequencies also show differences across epi-classes via the TAD query mode:

hicmaptools -in_map fly.bimap -in_bin fly.bins -TAD epiTAD. bed -output resTADs.tsv

HP1 TADs show a higher average intra-TAD contact density than the other three classes, whereas active TADs show the lowest contact density (Fig. 4, raw data in Additional file 3). This is in agreement with previous studies which reported that active chromatin domains present a weaker inside contact density than inactive domains, PcG, and null epiTADs [16].

\section{Running time benchmark}

Each query command generally completes within minutes. However, this varies based on the input scale, especially $\mathrm{HiC}$ maps. We conducted the following tests (Table 2) based on different input map resolutions and file formats on a personal desktop (4 GHz CPU, 32 GB memory).

The most time-consuming step of HiCmapTools is parsing the map and turning it into a hash structure, especially for text format. The running time increases from $6.23 \mathrm{~s}$ to $345.62 / 344.43 \mathrm{~s}$ when the map size increases from $67 \mathrm{MB}$ to $3.2 \mathrm{~GB}$ in text format. A text $\mathrm{HiC}$ map can be saved as a binary format via the genBinMap

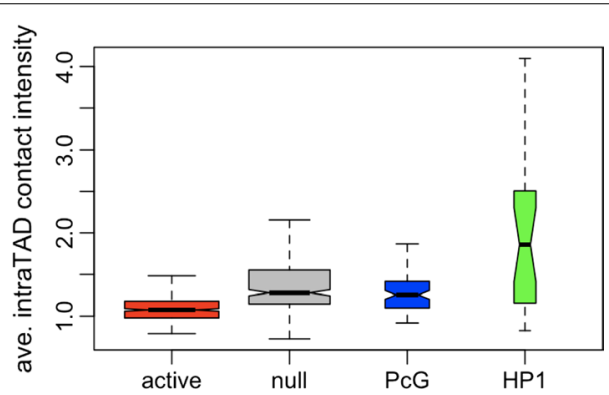

Fig. 4 Boxplot of average intra-TAD contact frequencies regarding epiTADs

Table 2 HiCmapTools runtimes for different query modes, map resolutions, and map formats of the fly map (dm3), where runtimes are recorded in user mode

\begin{tabular}{|c|c|c|c|c|c|c|}
\hline Resolution & Format & Size & Query & Per.Test & Time (sec) & ps \\
\hline $30 k$ & bin-contact/text & $67 \mathrm{MB}$ & bait & 100 & 6.23 & Section "bait query example" \\
\hline \multirow[t]{8}{*}{$1 \mathrm{k}$} & bin-contact/binary & $2.4 \mathrm{~GB}$ & loop & 0 & 11.34 & Section "TAD loop" \\
\hline & & & & 100 & 11.45 & \\
\hline & & & TAD & 0 & 11.32 & Section "TAD intra-density" \\
\hline & & & & 100 & 14.30 & \\
\hline & bin-contact/text & $3.2 \mathrm{~GB}$ & loop & 0 & 345.62 & \\
\hline & & & & 100 & 525.14 & \\
\hline & & & TAD & 0 & 344.43 & \\
\hline & & & & 100 & 525.54 & \\
\hline
\end{tabular}


command and loaded without parsing. This speeds up the running time by a factor of around $30(345.62 \rightarrow 11.34,344.43 \rightarrow 11.32)$.

\section{Conclusion}

We present a $\mathrm{C}++$ package that provides an efficient way to query $\mathrm{HiC}$ maps. $\mathrm{HiC}-$ mapTools supports seven access options so that biologists can quantify the contact frequency of the interest sites. Furthermore, the frequency probability is estimated based on a null hypothesis that shuffles the query position. Finally, the frequency is visualized as an output plot: a vertical line in the density plot of the random samples. The authors will continue to develop new functions for comparative $\mathrm{HiCs}$ to pursue $\mathrm{HiC}$ quantitative analysis.

\section{Availability and requirements}

- Project name: HiCmapTools

- Project home page: https://github.com/changlabtw/hicmaptools

- Project document page: https://hicmaptools.readthedocs.io/

- Operating system(s): platform-independent

- Programming language: $\mathrm{C}++$ and $\mathrm{R}$

- Other requirements: none

- License: GNU GPL

- Any restrictions to use by non-academics: license needed

\section{Abbreviation}

TAD: Topologicallyy Associating Domain.

\section{Supplementary Information}

The online version contains supplementary material available at https://doi.org/10.1186/s12859-022-04589-y.

Additional file 1. The list of epiTADs in bed format where the fourth column notes the epi-class: 1- active-red, 2null-gray, 3-PCG-blue and 4-HP1-green.

Additional file 2. Bash and R scripts for the experiment of "3.1 TAD loop".

Additional file 3. Bash and R scripts for the experiment of "3.2 TAD intra-density".

Acknowledgements

We thank Ming-Han Yang and Dr. Shih-Yu Chen for generously providing mouse HiCs used in this study. We thank the National Center for High-performance Computing (NCHC) for providing computational and storage resources. We thank two anonymous reviewers for their suggestions that improved the quality of the manuscript. In addition, we would like to acknowledge Dr. Aaron Heidel for his assistance in polishing the manuscript.

\section{Authors' contributions}

Conceived and designed the experiments: JMC GC. Contributed code: JMC YFW WTC FAL. Performed the experiments: JMC. Wrote the paper: JMC WTC GC. All authors have read and approved the manuscript.

\section{Funding}

This work was supported by the Taiwan Ministry of Science and Technology [106-2813-C-004-036-E to W-T. C. 108-2628-E-004-001-MY3 to J-M.C]. Research in the G.C. laboratory was supported by grants from the European Research Council (Advanced Grant 3DEpi, under grant agreement No. 788972), the European Union's Horizon 2020 Research and Innovation Programme (MuG, under grant agreement No. 676556), the Agence Nationale de la Recherche (ANR15-CE12-0006 EpiDevoMath), the Fondation pour la Recherche Médicale (DEI20151234396), the MSDAVENIR foundation 
(Project GENE-IGH), the INSERM, and the French National Cancer Institute (INCa). The funding bodies had no role in study design, data collection, experiment analysis, result interpretation, or manuscript preparation.

Availability of data and materials

The merged Drosophila embryo HiC contact map in bin-contact pair format: Bin: https://doi.org/10.6084/m9.figshare, 14730918. Contact: https://doi.org/10.6084/m9.figshare.6965576.

\section{Declarations}

\section{Ethics approval and consent to participate}

Not applicable.

\section{Consent for publication}

Not applicable.

\section{Competing interests}

The authors declare that they have no competing interests.

\section{Author details}

${ }^{1}$ Department of Computer Science, National Chengchi University, 11605 Taipei City, Taiwan. ${ }^{2}$ Institute of Human Genetics, CNRS and University of Montpellier, Montpellier, France.

Received: 6 September 2021 Accepted: 28 January 2022

Published online: 10 February 2022

\section{References}

1. Dekker J, Rippe K, Dekker M, Kleckner N. Capturing chromosome conformation. Science. 2002;295:1306-11.

2. Simonis M, Klous P, Splinter E, Moshkin Y, Willemsen R, de Wit E, et al. Nuclear organization of active and inactive chromatin domains uncovered by chromosome conformation capture-on-chip (4C). Nat Genet. 2006;38:1348-54.

3. Dostie J, Richmond TA, Arnaout RA, Selzer RR, Lee WL, Honan TA, et al. Chromosome Conformation Capture Carbon Copy (5C): a massively parallel solution for mapping interactions between genomic elements. Genome Res. 2006;16:1299-309.

4. Lieberman-Aiden E, van Berkum NL, Williams L, Imakaev M, Ragoczy T, Telling A, et al. Comprehensive mapping of long-range interactions reveals folding principles of the human genome. Science. 2009;326:289-93.

5. Forcato M, Nicoletti C, Pal K, Livi CM, Ferrari F, Bicciato S. Comparison of computational methods for Hi-C data analysis. Nat Methods. 2017;14:679-85.

6. Forcato M, Bicciato S. Capturing chromosome conformation, methods and protocols. Methods Mol Biol. 2020;2157:103-25.

7. Servant N, Varoquaux N, Lajoie BR, Viara E, Chen C-JJ, Vert J-PP, et al. HiC-Pro: an optimized and flexible pipeline for Hi-C data processing. Genome Biol. 2015;16:259.

8. Durand NC, Shamim MS, Machol I, Rao SS, Huntley MH, Lander ES, et al. Juicer provides a one-click system for analyzing loop-resolution Hi-C experiments. Cell Syst. 2016;3:95-8.

9. Robinson JT, Turner D, Durand NC, Thorvaldsdóttir H, Mesirov JP, Aiden EL. Juicebox.js provides a cloud-based visualization system for Hi-C data. Cell Syst. 2018;6:256-258.e1.

10. Kumar R, Sobhy H, Stenberg P, Lizana L. Genome contact map explorer: a platform for the comparison, interactive visualization and analysis of genome contact maps. Nucleic Acids Res. 2017;45:gkx644.

11. Yaffe E, Tanay A. Probabilistic modeling of Hi-C contact maps eliminates systematic biases to characterize global chromosomal architecture. Nat Genet. 2011;43:1059-65.

12. Yaffe E, Tanay A. hicpipe. 2012.

13. O'Sullivan JM, Tan-Wong SM, Morillon A, Lee B, Coles J, Mellor J, et al. Gene loops juxtapose promoters and terminators in yeast. Nat Genet. 2004;36:1014-8.

14. Sexton T, Yaffe E, Kenigsberg E, Bantignies F, Leblanc B, Hoichman M, et al. Three-dimensional folding and functional organization principles of the drosophila genome. Cell. 2012;148:458-72.

15. Dixon JR, Selvaraj S, Yue F, Kim A, Li Y, Shen Y, et al. Topological domains in mammalian genomes identified by analysis of chromatin interactions. Nature. 2012;485:376-80.

16. Szabo Q, Bantignies F, Cavalli G. Principles of genome folding into topologically associating domains. Sci Adv. 2019;5:eaaw1668.

17. Rao SS, Huntley MH, Durand NC, Stamenova EK, Bochkov ID, Robinson JT, et al. A 3D map of the human genome at kilobase resolution reveals principles of chromatin looping. Cell. 2014;159:1665-80.

18. Bonev B, Cohen N, Szabo Q, Fritsch L, Papadopoulos GL, Lubling Y, et al. Multiscale 3D genome rewiring during mouse neural development. Cell. 2017;171:557-572.e24.

19. Ogiyama Y, Schuettengruber B, Papadopoulos GL, Chang J-M, Cavalli G. Polycomb-dependent chromatin looping contributes to gene silencing during drosophila development. Mol Cell. 2018;71:73-88.e5.

20. Cheutin T, Cavalli G. The multiscale effects of polycomb mechanisms on 3D chromatin folding. Crit Rev Biochem Mol. 2019;54:1-19.

\section{Publisher's Note}

Springer Nature remains neutral with regard to jurisdictional claims in published maps and institutional affiliations. 